\title{
Los espacios públicos urbanos: lugares para el aprendizaje geográfico
}

\author{
Urban Public Spaces: Places for geographical Learning
}

\author{
Carmen Rosa Delgado Acosta ${ }^{*}$ y Carmen Gloria Calero Martíno**
}

Fecha de recepción: 19-05-2016 - Fecha de aceptación: 27-07-2016

Hábitat y Sociedad (ISSN 2173-125X), n. ${ }^{\circ}$ 9, noviembre de 2016, pp. 157-174.

\begin{abstract}
Summary
Urban public spaces display an interesting opportunity for teachers as places for learning. They are an excellent educational resource to accomplish programmed goals and competences in Geography for the Secondary and High school. Typological diversity of open public spaces allows analyzing not only elements of the structure and morphology in town, but also encouraged to reflect on the important role they represent for the whole society. We present a didactic proposal for the subject Geography and History at the Secondary School that includes a precise selection of the public spaces to analyses and the elaboration of specific worksheets, with the goal of exploring their role in organizing the urban fabric, environmental quality, security and accessibility; and in peoples' uses and practices. Additionally, this exercise will foster observational, cartographic and communicative skills, collaborative work, critical capacity and participation.
\end{abstract}

\section{Key words}

Public Spaces, Didactic Proposal, Geography, City, Urban Habitat, Education

\begin{abstract}
Resumen
Los espacios públicos abiertos ofrecen a los docentes una interesante oportunidad como lugares de aprendizaje. Constituyen un excelente recurso educativo en donde alcanzar gran parte de los objetivos y las competencias que la Geografía tiene programadas en la Educación Secundaria Obligatoria y el Bachillerato. La diversidad tipológica de los espacios públicos abiertos permite analizar no solo elementos de la estructura y la morfología urbanas sino que también anima a reflexionar sobre el importante papel que estos representan para el conjunto de la sociedad. La propuesta didáctica que se presenta para la asignatura de Geografía e Historia de la ESO pretende, mediante la correcta selección del espacio a analizar y a partir de fichas específicas de trabajo, estudiar el papel de los espacios públicos como organizadores de la trama urbana, su calidad ambiental, seguridad y accesibilidad, y los distintos usos y prácticas que, en ellos, llevan a cabo los ciudadanos. Asimismo, la actividad servirá para potenciar el desarrollo de las habilidades de observación, cartográficas y comunicativas, fomentará el trabajo colaborativo, la capacidad crítica y la participación.
\end{abstract}

\section{Palabras clave}

Espacio Público, Propuesta Didáctica, Geografía, Ciudad, Hábitat Urbano, Educación

\footnotetext{
* Doctora en Geografía y Catedrática de E. U. Departamento de Geografía e Historia, Facultad de Humanidades, Universidad de La Laguna (ULL). Correo: cdelgado@ull.edu.es.

** Doctora en Geografía y Catedrática de E. U. Departamento de Geografía e Historia, Facultad de Humanidades, Universidad de La Laguna (ULL). Correo: cgcalero@ull.edu.es.
} 


\section{Introducción}

Los espacios públicos ejercen una triple función: urbanística, ambiental y social. Son elementos estructurantes del tejido urbano, ordenadores de la trama, orientan el crecimiento y se convierten en nodos que facilitan la conectividad (Calero et al., 2014). Realizan una función ambiental y ecológica en la medida en que los espacios verdes mejoran el hábitat urbano, contribuyen a la oxigenación, a la regulación hídrica y ofrecen un ecosistema urbano apropiado para la conservación de la biodiversidad. Y, además, cumplen una función social, pues son lugares de encuentro, de integración y de intercambio; promueven la diversidad cultural y generacional de una sociedad; y generan valor simbólico, identidad y sentido de pertenencia (Borja y Muxí, 2000; Borja, 2003).

Estas cualidades convierten a los espacios públicos en un recurso muy valioso para el aprendizaje geográfico en la medida en que aúna elementos espaciales, ambientales, sociales y culturales que son los que conforman el espacio geográfico. Su utilidad didáctica se encuadra en un enfoque educativo que concede protagonismo al alumno, le permite desarrollar las competencias y reconoce la existencia de variados contextos de aprendizaje fuera del aula, escenarios reales en donde los estudiantes interactúan con el entorno.

El aprendizaje experimental como método de enseñanza se conoce desde principio del siglo XX y tiene como finalidad promover una enseñanza más amena, motivadora, participativa y vinculada con el medio, superando las enseñanzas de tipo reproductivo y memorístico, donde no está presente la participación activa de los alumnos. En la enseñanza de la Geografía, el uso de contextos educativos de enseñanzaaprendizaje externos al aula ha sido, tradicionalmente, una estrategia muy valorada por las diversas corrientes didácticas, reconociéndose la salida de campo como una actividad pedagógica relevante que favorece la enseñanza por parte del docente y el aprendizaje significativo de los estudiantes.

En los momentos actuales en el que las nuevas tecnologías acaparan casi todas las propuestas didácticas innovadoras y el aprendizaje en los espacios virtuales se ha convertido en el paradigma, casi único, de las metodologías de enseñanza, conviene rescatar el aprendizaje fuera del aula por las posibilidades que ofrece como recurso didáctico imprescindible para el enfoque de la Geografía Activa y como parte esencial de un aprendizaje constructivo. El contacto con la realidad, la observación, la experimentación directa, son formas de enseñanza habituales en la Geografía escolar, que no deberían permanecer postergadas en pro de las nuevas tecnologías que usan el ordenador e internet como únicas herramientas; muy al contrario son estrategias que se complementan permitiendo una mejor consecución de los objetivos de las materias con contenidos geográficos y las competencias clave de etapa.

El artículo se estructura en tres apartados: en el primero se repasa el concepto de espacio público y se analiza cuál ha sido su uso como recurso educativo; se aprovecha para reflexionar sobre el aprendizaje fuera del aula como método de enseñanza y de aprendizaje para la ciencia geográfica. En el segundo apartado se analiza el currículo de la Educación Secundaria Obligatoria (ESO) con la finalidad de constatar las cuestiones geográficas de este nivel educativo que son susceptibles de estudiarse directamente en los espacios públicos abiertos. Y por último, se proporciona una propuesta didáctica en donde se concreta 
la manera de llevar a cabo la enseñanza de los diversos aspectos a estudiar, y donde el alumno será protagonista de su propio aprendizaje.

\section{Los espacios públicos abiertos como espacios de aprendizaje fuera del aula}

Cada materia cuenta con sus propias herramientas de enseñanzaaprendizaje y el trabajo de campo, el realizado fuera del aula, se ha considerado una de las técnicas educativas más eficaces para enseñar Geografía desde su inclusión como disciplina básica en los estudios no universitarios (Montilla Pacheco, 2005, Godoy y Sánchez, 2007; Souto, 1998). Las diversas corrientes didácticas de la Geografía, desde sus específicas formas de afrontar los procesos de enseñanza-aprendizaje, han valorado la viabilidad y vigencia del contacto directo con el espacio geográfico como el mejor método para que los conocimientos adquieran significatividad para el alumnado (Lossio, 2004, citado por Godoy y Sánchez, 2007; García de la Vega, 2008). Sin embargo, para que el aprendizaje en el entorno fuera del aula adquiera relevancia como estrategia docente, es necesario que se inserte en una metodología de enseñanza motivadora (Palacios, 1988; Rodríguez, 2006), que implique al alumno en el proceso de adquisición de las competencias programadas y no sea una mera herramienta puntual aplicada en contextos de enseñanza tradicionales.

La renovación pedagógica y didáctica que se está viviendo actualmente, ocasionada tanto por los cambios que se han producido en las vías para la adquisición de los conocimientos ante el auge de las nuevas tecnologías, como por las instrucciones o directivas europeas e internacionales sobre el futuro de la educación, supone la utilización de metodologías de enseñanza centradas en el alumno que enfaticen los procesos en la adquisición de las competencias. La Ley Orgánica 2/2006 de Educación incorporó las competencias básicas al currículo, como uno más de sus elementos, y les otorgó el papel de referente tanto para la evaluación como para el diagnóstico. Con esta decisión legislativa, se asumieron en España las conclusiones de los Organismos internaciones (la OCDE y la Comisión Europea) y del Programa para la evaluación internacional de los alumnos de la propia OCDE (PISA) sobre la enseñanza y aprendizaje de las competencias básicas como un medio para mejorar la calidad y la equidad del sistema educativo. Precisamente una de las principales aportaciones de la Ley Orgánica de Educación de 2006 (LOE) fue la inclusión de las competencias básicas en el currículo de la enseñanza obligatoria, concebidas como la capacidad de poner en marcha, de forma integrada, los conocimientos y destrezas adquiridos y los rasgos de personalidad que permitan resolver situaciones diversas. Requiere, por tanto, la utilización de recursos variados, no solo del saber y del saber-hacer sino de también de saber ser y estar, aplicables en contextos diversos

La Ley Orgánica 8/2013, de 9 de diciembre para la Mejora de la Calidad Educativa (LOMCE) mantiene las competencias del currículo y las extiende a todos los niveles no universitarios, pues las orientaciones de la Unión Europea insisten en la necesidad de la adquisición de las competencias clave por parte de la ciudadanía como condición indispensable para lograr que los individuos alcancen un pleno desarrollo personal, social y profesional que se ajuste a las demandas de un mundo globalizado y haga posible el desarrollo económico, vinculado al co- 
nocimiento. Asimismo, reconoce que para potenciar la motivación por el aprendizaje de competencias se requieren, además, metodologías activas y contextualizadas que faciliten la participación del alumnado. De forma que los métodos de enseñanza recomendados, incluso por la propia legislación educativa actual, son los que permiten la adquisición de los conocimientos a partir de situaciones reales (Ministerio de Educación, Ciencia y Cultura, 2015b).

La educación experimental que proporciona al alumno la posibilidad de aprender de forma activa — recopilando, organizando y mostrando la información, y planteando preguntas y soluciones a los problemas detectados- no solo cuenta con el aula para desarrollar su metodología, antes bien debe apoyarse en otros contextos educativos fuera de ella (Trilla, 2006), muy adecuados para el logro de las competencias programadas (Molina, 2007). Sin embargo, y a pesar del reconocimiento incuestionable de las salidas de campo, pocos son los proyectos de innovación educativa actuales que plantean la utilización de estrategias didácticas en contextos ajenos al aula. Si nos detenemos a analizarlos —basta una exploración a los proyectos de innovación de las distintas Consejerías de Educación- en su mayor parte, van encaminados a lograr el aprendizaje activo y exploratorio a partir de actividades que hacen uso de las nuevas tecnologías (EarthQuest, GeoQuest, WebQuest, MiniQuest, CazaTesoros, etc.).

Sin restarle valor a las TIC como herramientas que proporcionan grandes ventajas educativas - motivación, trabajo autónomo, alfabetización digital, acceso rápido a una gran cantidad de información, etc.- , no hay que dejar en el olvido el contacto directo del alumno con el entorno, pues es, precisamente ahora, en el marco de los cambios metodológicos actuales, cuando cobra especial relevancia convirtiéndose en una de las principales estrategias de la didáctica de la Geografía para la consecución de los objetivos y de las competencias (García de la Vega, 2008).

Tradicionalmente para la Geografía escolar — junto con los paisajes naturales- la ciudad ha sido un lugar para el aprendizaje geográfico y los itinerarios urbanos, al poner en contacto al alumnado con la realidad, permiten desarrollar los conocimientos adquiridos en el aula, fijar los conceptos, ofrecerles explicaciones más consolidadas de los hechos geográficos y despertarles el espíritu crítico ante la pertinencia de las transformaciones antrópicas. Pero el uso didáctico del paisaje urbano se ha centrado en dar a conocer al alumno la morfología y la estructura urbana, poniendo el énfasis en determinados aspectos según sea la perspectiva geográfica utilizada - la confección de los mapas mentales en la Geografía de la Percepción, la producción del espacio urbano en la Geografía Crítica, etc.

La idea de que las ciudades educan es antigua, las primeras reflexiones corresponden al informe de la UNESCO de 1972 titulado Aprender a ser de E. Faurer, aunque fue en la década de los ochenta cuando se instaló con fuerza la expresión de "ciudad educadora" en los discursos pedagógicos. En 1990 en el I Congreso Internacional de ciudades educadoras celebrado en Barcelona, se plantearon las tres dimensiones de la ciudad educadora: la ciudad como contenedor de recursos educativos (aprender en la ciudad); como agente educativo (aprender de la ciudad); y la ciudad como contenido u objetivo educativo (aprender la ciudad) (Trilla, 2006, p. 22).

Dentro de este amplio y rico abanico de posibilidades didácticas que la ciudad nos ofrece, uno de los contextos urbanos de mayor potencia- 
lidad educativa y, hasta el momento, poco explorado en la enseñanza de la Geografía son los espacios públicos, en tanto que constituyen un elemento esencial de las ciudades al actuar simultáneamente como organizadores de la estructura urbana y como lugares de encuentro e intercambio social y cultural.

El concepto de espacio público permite acepciones diversas según las distintas corrientes de pensamiento pero, en este caso, nos referimos a los espacios exteriores y abiertos - parques, plazas, plazoletas-, de acceso libre, que se encuentran fuera de la influencia del consumo, y a los que acude gran diversidad de personas para realizar actividades variadas. Son los lugares donde se produce la interrelación, el encuentro social y el intercambio (García Ramón et al., 2004), de ahí su importancia como espacios de socialización (Perahia, 2007).

En relación a las posibilidades educativas de los espacios públicos, la investigación realizada por Enriqueta Molina Ruiz ${ }^{1}$ (2007) referida exclusivamente a los parques y jardines, ha puesto de manifiesto que a pesar de que la mayoría del profesorado los considera contextos formativos interesantes, no suelen valorarlos como recursos generadores de aprendizajes significativos integrados en el currículo; todo lo más, los consideran espacios lúdicos en donde desarrollar actividades extracurriculares.

Sin embargo, las potenciales pedagógico-didácticas de los espacios públicos para la consecución de las competencias, tanto de la Educación Primaria como de la Secundaria Obligatoria, son muchas y variadas, pues no solo facilitan el logro de competencias de carácter actitudinal —valores, actitudes y comportamientos como el respeto al medio y a las personas, la habilidad para relacionarse, la capacidad para despertar la curiosidad, la exploración, etc.- , sino que permiten adquirir otras muchas competencias, así como la aplicación de los conocimientos y la puesta en práctica de proyectos de estudio de la mayor parte de las materias curriculares. Las que, a priori, parecen más proclives a su utilización como recurso didáctico, según el sentir del profesorado (Molina, 2007, p. 11), son las Ciencias de la Naturaleza y la Educación Artística; en cambio, la Geografía no parece reclamarlos en igual medida.

Por su parte, la escasa literatura existente sobre los espacios públicos como lugares de aprendizaje centran su interés, básicamente, en su utilización para la Educación Ambiental (Benayas et al., 1999) y para el despertar de la conciencia cívica (Páramo, 2010; Ucar, 2006), contenidos que se encuadran en la materia de Educación Cívica y constitucional ${ }^{2}$ de la LOMCE.

En su mayor parte no se trata de proyectos de innovación educativa - hay algunas iniciativas minoritarias en este sentido-, sino de estudios que muestran las posibilidades de los parques y espacios públicos urbanos para el aprendizaje de determinados aspectos educativos.

En relación a la Educación Ambiental, hay un reconocimiento bastante generalizado de la función didáctica que poseen los espacios verdes y parques urbanos por la gran riqueza biológica que encierran - diferentes estratos vegetales, zonas húmedas, refugio de variadas especies animales, etc.- de ahí que para muchos de ellos los gestores públicos editen guías descriptivas ${ }^{3}$ sobre los aspectos más representativos. Suelen ser documentos ajenos a los objetivos de la Educación Ambiental, pues se trata de una mera trasmisión estática de conocimientos —vegetación y avifauna, principalmente-, pero no se conciencia sobre los problemas ambientales ni se proporciona una visión crítica
1 La autora centró su investigación en averiguar las posibilidades didáctico-pedagógicas y formativas de los parques y jardines, recabando la opinión del profesorado no solo sobre su valoración como contexto educativo, sino con qué finalidad puede ser utilizado, qué potencialidades formativas poseen, qué estrategias metodológicas permiten aplicar, y qué materias son más susceptibles de utilizarlos como recurso educativo.

2 En este sentido, ha existido una interesante reflexión desde la Geografía, donde se formulan las posibles relaciones entre esta disciplina de larga tradición educativa y la formación de los ciudadanos (AGE, 2007), que demuestra la capacidad de esta materia para conocer y analizar críticamente las relaciones entre las sociedades y los territorios y de reflexionar sobre las percepciones espaciales, las desigualdades sociales, las diferencias de género, la sensibilidad hacia la conservación del patrimonio, etc.

3 Benayas del Álamo, Gutiérrez Mora y Gutiérrez Pérez (1999) ponen de manifiesto que las Comunidades Autónomas de Zaragoza, Madrid y Cataluña son las que han generado en mayor medida este tipo de materiales, en cambio en otras - como por ejemplo la Canaria—, los gestores públicos no parecen haber tenido interés por fomentar la vertiente educativa de los parques. 
de los inconvenientes por los que suelen atravesar estos espacios verdes (Benayas, et al., 1999:65).

De igual manera, los espacios públicos urbanos, aun siendo escasamente utilizados, son muy valorados como contextos de aprendizaje fuera del aula, muy adecuados para la educación de comportamientos cívicos que contribuyen a la convivencia ciudadana; escenarios donde se pueden aprender las prácticas sociales y sirven también para identificar y cohesionar los diversos grupos culturales (Páramo, 2010, p. 131; Grupo de Pedagogía Urbana y Ambiental, 2008). Algunas competencias clave - sociales y cívicas y aprender a aprender- desarrolladas en la asignatura de la Educación Cívica y constitucional pueden ser trabajados en los parques y plazas públicas - respeto por las costumbres y modos de vida de las personas; conocer y apreciar los valores y normas de convivencia y obrar de acuerdo con ellas; desarrollar habilidades emocionales comunicativas y sociales para actuar con autonomía en la vida cotidiana y participar activamente en las relaciones de grupo mostrando actitudes generosas y constructivas-. En definitiva, como lugares de encuentro de gran diversidad de personas, se convierten en lugares idóneos en los que aprender las pautas de conducta para una convivencia cívica.

Aunque sean estas dos las materias donde ha habido más interés por el uso didáctico del espacio público urbano, no cabe duda de que son muchas otras las que podrían verse beneficiadas, como es el caso de la Educación Artística - lugar de inspiración para el dibujo y la pintura, estudio de expresiones artísticas diversas entre las que destaca la escultura, los murales, las exposiciones de arte, etc.-—; la Educación Física puede aprovechar también las variadas dotaciones de equipamientos deportivos que suelen tener muchos parques - canchas de baloncesto, lugares para el skate, las tirolinas, escalada, etc.- - que, si bien están pensadas para actividades deportivas informales, podrían ser objeto de proyectos educativos escolares.

Asimismo, las Ciencias Sociales, y en especial la Geografía, también encuentran en el espacio público un contexto educativo inmejorable en donde los objetivos y las competencias clave programadas para la etapa de la ESO pueden verse reforzados.

\section{Aprender Geografía en ESO a través del espacio público}

La Educación Secundaria Obligatoria constituye el segundo segmento de la Enseñanza Obligatoria y comprende un periodo extenso y decisivo no solo en la adquisición de nuevos contenidos y en el aprendizaje y práctica de habilidades diversas sino, de forma especial, en el desarrollo de la persona, marcado por el tránsito de la infancia a la juventud, lo que implica que los escolares muestran nuevas actitudes y demandas, al tiempo que ese mismo desarrollo personal permite diversificar y ampliar el proceso de aprendizaje desarrollando nuevas metodologías no exploradas en la etapa anterior.

Se trata de una educación común cuyos objetivos van claramente dirigidos a lograr que los alumnos adquieran capacidades relacionadas con los tres ámbitos: el saber, el saber hacer y el saber ser y estar. También la ESO se presenta como un periodo educativo con vocación orientadora y no como un simple tránsito al Bachillerato y la formación universitaria.

La incorporación de las competencias al currículo es, pues, una pri- 
mera apuesta por acercar el sistema educativo español a las exigencias internacionales. De siete competencias clave que mantiene la LOM$\mathrm{CE}$ en la Enseñanza Secundaria Obligatoria, hay dos directamente relacionadas con la materia de Geografía e Historia: la sociales y cívicas y conciencia y expresiones culturales. Ambas pretenden fomentar en el alumno una gran variedad de conocimientos, destrezas y actitudes como la percepción y el conocimiento del espacio en que se desarrolla la actividad humana; la comprensión del espacio en que tienen lugar los hechos sociales y la propia vida del alumno; los procedimientos de orientación, localización, observación e interpretación de los espacios, paisajes, reales o representados; el conocimiento de la interacción hombre-medio y la organización del territorio resultante; el análisis de la acción de los seres humanos en la utilización del espacio y de sus recursos; y las habilidades para asegurar la protección y el cuidado del medio ambiente. Por tanto, una gran cantidad de recursos que el alumno debe aprender a emplear y para los que hay que utilizar metodologías y estrategias de enseñanza-aprendizaje variadas, entre las que destaca por su eficacia en la consecución de dichas competencias, la educación experimental y el contacto directo del alumno con el entorno.

La inclusión de las competencias clave en el currículo permite identificar aquellos aprendizajes que se consideran imprescindibles para integrarse en la sociedad de modo crítico, contribuyendo a una formación que permita ejercitar los derechos y obligaciones ciudadanos, y a la adquisición de los elementos básicos de la cultura para así conocer e interpretar el medio que rodea a los alumnos y alumnas y, sobre todo, seguir aprendiendo de manera autónoma a lo largo de la vida. Esto no significa que deba existir una relación directa entre las diversas materias y cada una de las competencias, al contrario, las distintas competencias se trabajan en las distintas materias en mayor o menor grado y algunas de ellas, como ya se ha señalado, se alcanzan con mayor facilidad en algunas áreas específicas que, por sus propios objetivos y por la naturaleza de sus contenidos, lo favorecen.

Comprender la vinculación que existe entre los objetivos y las competencias constituye un reto para los profesores que deben entender que el nivel de las competencias está referido a aquellos contenidos, habilidades y actitudes indispensables, que los escolares deben extraer en cada uno de los periodos educativos y que les va a permitir un adecuado desarrollo personal; de forma que se puedan ir incorporando a la vida adulta con plenas capacidades y, además, sepan continuar aprendiendo a lo largo de la vida. En la consecución de las competencias interviene todo, es decir, cualquier aprendizaje de cualquiera de las materias, puede y debe estar orientado a la consecución de una o varias de las competencias de forma explícita o implícitamente. De esta forma, los profesores deben tener presentes en sus programaciones qué competencias se trabajan en cada una de las unidades didácticas y a través de qué tipo de actividades se van a potenciar.

Sin embargo existen determinadas acciones educativas singulares y específicas que pueden permitir concentrar el trabajo de varias o incluso de la totalidad de las competencias clave de la etapa. En este sentido, las actividades de carácter experimental que permiten a los alumnos aprendizajes activos son el recurso idóneo para desarrollar y/o reforzar el nivel de competencias que los alumnos han ido adquiriendo a lo largo de un periodo.

La Geografía en la Educación Secundaria Obligatoria constituye 
uno de los ejes vertebradores de la materia Geografía e Historia. Una parte de los contenidos geográficos que se desarrollan en esta etapa ya han sido esbozados en la Educación Primaria en el área de Ciencias Sociales, con una organización diferente, y un nivel y desarrollo que se adecua a la edad de los escolares, centrado principalmente en su entorno inmediato. Sin embargo, las características de los alumnos de Secundaria permiten, por una parte, una mayor desagregación curricular y hacen posible un enfoque más disciplinar, aunque conviene tener presente que el desarrollo intelectual del alumnado hace imprescindible un trabajo gradual para que los escolares vayan asimilando la complejidad del análisis espacial, la relación entre el territorio y la sociedad que lo habita, al tiempo que procesan informaciones variadas, a diferentes escalas.

Amparados por los objetivos generales de la etapa, los objetivos propios de la materia van encaminados al aprendizaje de todos aquellos aspectos relacionados con la adquisición de conocimientos, actitudes y habilidades relacionados con las Ciencias Sociales en general y de forma específica con la Geografía y la Historia. El conocimiento geográfico aparece bien diferenciado y graduado: a lo largo del Primer Ciclo de la Secundaria se organizan los contenidos geográficos articulados en dos grandes bloques: el medio físico y el espacio humano que desarrollan los conceptos generales y los aplican en diferentes escalas: España, Europa y el mundo. En $4 .^{\circ}$ curso se analizan los aspectos geográficos más destacados en relación con la globalización.

A pesar de presentar una organización tradicional del aprendizaje, el currículo de Geografía ha ido introduciendo, en los últimos años, contenidos innovadores que dotan a los programas de mayor actualidad y pueden permitir que alumnos y profesores analicen hechos y fenómenos de carácter y/o consecuencias espaciales que se están produciendo en el momento actual y de importantes repercusiones en las sociedades y los territorios. Así, los problemas de conservación del medio natural, los riesgos naturales, las principales amenazas medioambientales, las desigualdades en el desarrollo, los desequilibrios poblacionales originados por las corrientes migratorias, las sociedades multiculturales resultantes, las transformaciones que ha proporcionado la globalización, etc., son algunas de las propuestas que la Geografía plantea en la escuela, lo que invita a pensar en el dinamismo de la ciencia geográfica que ha abordado, en las dos últimas décadas, un buen número de contenidos variados centrándose en la perspectiva espacial y en sus consecuencias territoriales y sociales.

Como ya se ha señalado, los espacios públicos abiertos constituyen un contexto de aprendizaje no demasiado explorado desde la Didáctica de la Geografía, quizás porque su vinculación con los contenidos disciplinares podría no parecer muy clara para los docentes. Sin embargo muchos de los temas que se abordan a lo largo de la ESO en la asignatura de Geografía e Historia se pueden enlazar con ellos de forma directa o indirecta.

Es evidente que los espacios públicos abiertos deben ser trabajados cuando se aborda el estudio de la ciudad ya que constituyen una parte esencial de la estructura urbana, jerarquizan el espacio, generan centralidades y son el soporte de muchas de las funciones que la propia ciudad desarrolla. También son el escenario de gran parte de la vida de los ciudadanos y ejemplifican, en muchos casos, los problemas principales de las sociedades urbanas. Los espacios públicos abiertos son, en general, piezas clave de la historia de la ciudad y constituyen hitos del 
desarrollo urbano, a veces esponjan el caserío, otras lo incitan a crecer, lo transforman y le dan personalidad propia, pero siempre ayudan a explicar el modo de crecimiento y suelen ser los referentes de etapas singulares. Los espacios públicos abiertos constituyen, en numerosos casos, lugares simbólicos de la ciudad, donde se reúnen y mezclan las edificaciones más relevantes, de mayor interés artístico o simplemente cultural, político o social. En otros casos, son simplemente los lugares centrales de barrios o distritos, zonas de ocio y de intercambio social, auténticos desahogos urbanos, donde la vida de los ciudadanos se extiende más allá de sus viviendas.

La diversidad tipológica de los espacio públicos abiertos que se pueden abordar en cualquier actividad didáctica relacionada con el estudio de la ciudad, permite analizar no solo elementos de la estructura y la morfología urbanas, sino también anima a pensar sobre una parte importante de las funciones urbanas que, en muchos casos, se desarrollan en el entorno de estos espacios o, en muchas ocasiones, en ellos mismos, que se convierten en auténticos contenedores de actividades diversas.

También en los espacios públicos abiertos se evidencia la estructura de la población, la diversidad cultural, las desigualdades y los conflictos. La necesidad de espacios públicos para acoger a una población cada vez más envejecida que reclama lugares de recreo y descanso, la apropiación de estos espacios por población inmigrante, la presencia de otros grupos como los niños y las mujeres, permiten realizar observaciones diversas y distintas que nos remiten a la Geografía de forma inequívoca y que enlazan con una gran parte de los temas que desarrolla el currículo de la ESO.

La sostenibilidad urbana, la calidad medioambiental de las ciudades, el desarrollo de determinadas actividades económicas que utilizan el espacio público como soporte o se desarrollan en su entorno, el conocimiento del patrimonio artístico que muchos de ellos contienen, la movilidad de los ciudadanos, la apropiación de estos lugares por minorías, las demandas de los diferentes usuarios, son otros aspectos que permiten análisis combinados y sustanciosos.

El trabajo en los espacios públicos no solo permite adquirir conocimientos y relaciones sobre la propia ciudad y la población que la vive, sino que desarrolla destrezas propias del campo de la Geografía y de otras ciencias sociales: la lectura de mapas y planos, la comprensión de las diferentes escalas, la observación y la recogida sistemática de datos, la encuesta y su tratamiento, la explicación de imágenes, en definitiva, un conjunto amplio de habilidades que refuerzan el aprendizaje y generan nuevas posibilidades y aplicaciones en cualquier otro campo educativo.

Todos estos aspectos nos remiten a la utilidad que el trabajo en cualquier espacio público puede tener para los escolares, en una etapa de sus vidas de constante transformación, de apertura y mayor libertad, en la que el uso y disfrute del espacio público se puede convertir además en motivo de aprendizaje personal.

\section{Diseño de una propuesta didáctica}

La utilización didáctica de los espacios públicos urbanos en el proceso de enseñanza-aprendizaje de la Geografía se puede desarrollar en la materia de Geografía e Historia de la Educación Secundaria Obligatoria, en 
concreto, en los contenidos referidos a las ciudades y al proceso de urbanización que se imparte en el primer ciclo de esa etapa educativa. También podría ser pertinente en la asignatura de Geografía de segundo de Bachillerato, pero, en este caso, habría que adaptar cualquier propuesta a los presupuestos curriculares de la etapa. De forma tangencial, diversos aspectos relacionados con la población, la estructura por edad y género y los movimientos migratorios pueden reforzarse a través del desarrollo de cualquier actividad relacionada con los espacios públicos.

La propuesta didáctica debe contener sus propios objetivos específicos. Estos objetivos irán encaminados a que los estudiantes reconozcan que los espacios públicos existen en todas las ciudades y suelen ser elementos organizadores de la trama urbana, por lo que se localizan en todo el plano urbano: centro histórico, ensanche, y periferia. Y que, en su mayor parte, son el resultado de una planificación previa, es decir, se proyectan con unos determinados objetivos y presentan diferentes diseños que deben ser convenientemente identificados: unos son parques, otros son plazas, paseos o zonas peatonales. También resulta conveniente aprender a identificar los elementos principales que están presentes en la mayor parte de los espacios públicos abiertos: el mobiliario urbano básico — bancos, papeleras...- - y el más específico —elementos para el juego de los niños, para la realización de ejercicios de carácter deportivo...-, la pavimentación, el alumbrado, la existencia de agua, la vegetación, los elementos ornamentales y artísticos... Todo ello debe ser observado y evaluado por los escolares y va a permitir valorar la funcionalidad, la capacidad de usos, la calidad ambiental, la seguridad, la accesibilidad y otros aspectos derivados del equipamiento del espacio en estudio; además ayuda a potenciar la capacidad propositiva y el concepto de que los espacios públicos, como lugares colectivos, pueden ser transformados y mejorados y se deben adaptar a las necesidades y aspiraciones de los ciudadanos.

La propuesta intentará que el alumnado comprenda que los espacios públicos están destinados para el uso de todos los ciudadanos por lo que son los lugares de encuentro y de socialización de personas de diferentes edades, sexos y nacionalidades, que los utilizan para llevar a cabo en ellos actividades diversas que suelen ser diferentes según el grupo de edad y sexo (pasear, jugar, descansar, leer, etc.), o que pueden convertirse en lugares de encuentro de personas con los mismos intereses, incluso dificultades, y a que encuentran en ellos el lugar para la reunión, la reivindicación y, en ocasiones, la estancia permanente. Reflexionar sobre el espacio público como un bien común, como una parte de la ciudad que es de todos los ciudadanos y que debe ser respetado y que exige un esfuerzo colectivo para su correcta conservación, debe presidir el análisis de cualquier espacio en estudio.

Para llegar a la consecución de estos objetivos, la propuesta estimulará el desarrollo de habilidades de observación, cartográficas y comunicativas, fomentará el trabajo colaborativo, la capacidad crítica y la participación.

\section{Objetivos y competencias clave}

Una propuesta didáctica que trabaje uno o varios espacios públicos no debe plantearse de forma aislada sino que tiene que contribuir a desarrollar los objetivos de la etapa y aquellos que se programen para los contenidos relativos a la ciudad y el proceso de urbanización a diferentes escalas. 
En cuanto a los objetivos de la etapa, la propuesta de trabajo en el espacio público permite a los alumnos y las alumnas conocer y valorar estos lugares como espacio colectivos, que, aparte de su importancia como elementos físicos indispensables en la construcción y el desarrollo de la ciudad, son lugares de encuentro, de tránsito, de reunión de grupos diversos, donde coinciden personas diferentes a las que asiste el derecho a participar libremente de la vida urbana. También, en ellos, la diversidad de usuarios anima a poner en valor el respeto por la diferencia entre los sexos, las edades y cualquier otra condición personal. Son sitios de reivindicación, de ocio, de desarrollo de múltiples actividades que enseñan a comprender una sociedad plural y democrática. Muchos de ellos son lugares patrimoniales, simbólicos o contienen elementos artísticos que pueden ser reconocidos y que es necesario proteger y conservar. Un trabajo bien orientado en cualquier espacio público puede favorecer actitudes de sociabilidad, permite rechazar la discriminación y proporciona herramientas al alumnado para comprender mejor el correcto funcionamiento de una sociedad democrática y solucionar conflictos. Desde el punto de vista metodológico, la propuesta estimula el desarrollo de destrezas básicas en la utilización de fuentes de información, alienta el sentido crítico y la participación. La capacidad para tomar decisiones, aprender a aprender y planificar, asumiendo responsabilidades son otros aspectos que pueden trabajarse y que conectan de forma directa con los objetivos de la etapa secundaria. Prácticamente todos los objetivos de la ESO pueden trabajarse en los espacios públicos:

a) Asumir responsablemente sus deberes, conocer y ejercer sus derechos en el respeto a los demás, practicar la tolerancia, la cooperación y la solidaridad entre las personas y grupos, ejercitarse en el diálogo afianzando los derechos humanos y la igualdad de trato y de oportunidades entre mujeres y hombres, como valores comunes de una sociedad plural y prepararse para el ejercicio de la ciudadanía democrática.

b) Desarrollar y consolidar hábitos de disciplina, estudio y trabajo individual y en equipo como condición necesaria para una realización eficaz de las tareas del aprendizaje y como medio de desarrollo personal.

c) Valorar y respetar la diferencia de sexos y la igualdad de derechos y oportunidades entre ellos. Rechazar la discriminación de las personas por razón de sexo o por cualquier otra condición o circunstancia personal o social. Rechazar los estereotipos que supongan discriminación entre hombres y mujeres, así como cualquier manifestación de violencia contra la mujer.

d) Fortalecer sus capacidades afectivas en todos los ámbitos de la personalidad y en sus relaciones con los demás, así como rechazar la violencia, los prejuicios de cualquier tipo, los comportamientos sexistas y resolver pacíficamente los conflictos.

e) Desarrollar destrezas básicas en la utilización de las fuentes de información para, con sentido crítico, adquirir nuevos conocimientos. Adquirir una preparación básica en el campo de las tecnologías, especialmente las de la información y la comunicación.

f) Concebir el conocimiento científico como un saber integrado, que se estructura en distintas disciplinas, así como conocer y aplicar los métodos para identificar los problemas en los diversos campos del conocimiento y de la experiencia. 
g) Desarrollar el espíritu emprendedor y la confianza en sí mismo, la participación, el sentido crítico, la iniciativa personal y la capacidad para aprender a aprender, planificar, tomar decisiones y asumir responsabilidades.

h) Comprender y expresar con corrección, oralmente y por escrito, en la lengua castellana y, si la hubiere, en la lengua cooficial de la Comunidad Autónoma, textos y mensajes complejos, e iniciarse en el conocimiento, la lectura y el estudio de la literatura.

i) Conocer, valorar y respetar los aspectos básicos de la cultura y la historia propias y de los demás, así como el patrimonio artístico y cultural (Ministerio de Educación, Ciencias y Cultura, 2015a).

En relación con las competencias clave, el reconocimiento del espacio público como un lugar de socialización y la potenciación de su uso correcto, ayudará a aprender a convivir en una sociedad democrática respetando a los demás y a ejercitarse en habilidades de valoración, de respeto, de empatía e igualdad de género. Todo ello refuerza las competencias sociales y cívicas. La preparación previa que exige el reconocimiento de la zona a estudiar obliga a los estudiantes a utilizar la cartografía digital. El vaciado de las fichas de observación y su posterior tratamiento, la presentación de los resultados en Power-Point, y la búsqueda de información en páginas web sobre las características de los espacios públicos les ayudará a mejorar la competencia digital. El progreso en la comunicación lingüistica se consigue a partir de las todas las tareas que se proponen: ampliación del vocabulario específico, lectura de planos, comentario de gráficos, exposición de resultados y debates. La observación del espacio, el tratamiento de la información recogida y su comunicación propician el auto aprendizaje (aprender a aprender). El sentido de la iniciativa se potencia mediante la organización autónoma del trabajo en los grupos y la búsqueda de soluciones a los problemas detectados, ayuda a ser responsables de la planificación de las tareas y fomenta la creatividad. La presencia de manifestaciones artísticas diversas o el carácter histórico y simbólico de algunos de estos lugares propician el cultivo estético y la sensibilidad del alumnado que profundizará en la competencia de conciencia y expresiones culturales. De forma que cinco de las siete competencias ${ }^{4}$ clave de la ESO pueden trabajarse a partir de los espacios públicos.

\section{Estructura de la propuesta didáctica}

\section{Fase de reflexión-preparación}

Antes de llevar a cabo la actividad corresponde al profesor/a elegir el espacio a estudiar. Dependiendo del número de alumnos se podrán elegir varios espacios con la finalidad de comparar sus características - lo que enriquecería notablemente el conocimiento sobre los procesos de crecimiento urbano y las diversas tipologías - o bien elegir un solo espacio, de forma que la investigación se segmente y concurra al final con las conclusiones de cada uno de los grupos.

En cualquiera de los casos, el espacio público debe elegirse atendiendo a los siguientes criterios:

a) Espacios públicos de gran vitalidad y simbolismo. Con carácter de centralidad dentro del núcleo urbano.

b) Espacios públicos con gran intensidad de uso y diversidad de 
usuarios que puedan ser centrales a escala de toda la ciudad o de alguno de sus distritos o barrios.

a) Espacios públicos que conciten actividades de uso distintas y bien diferenciadas tanto a escala de toda la ciudad como de alguno de sus barrios.

Modalidades de espacios a estudiar:

a. Espacio público central del barrio cercano al centro educativo (plaza, rambla). Esta elección permite:

- Facilidad para realizar el trabajo de campo.

- Posibilidad de un conocimiento previo del alumnado sobre el espacio. Experiencias propias sobre los cambios en el aspecto formal del espacio y en los usos.

b. Espacio público central y simbólico de la ciudad en donde se ubica el centro. Esta elección permite:

- Identificación de los elementos que han convertido ese espacio en un lugar central y simbólico.

- Posibilidad de experiencia de ciertos usos por parte del alumnado.

- Descubrimiento de la vitalidad de espacio en usos, usuarios diversos y actividades distintas que se realizan.

c. Espacio público de nueva creación. Puede ser un espacio renovado del centro urbano o de nueva creación en la periferia. Esta elección permite:

- Apreciación del dinamismo urbano; la ciudad es un ente vivo que se remodela y crece, y, con ella, el espacio público.

- Identificación de los objetivos de creación del espacio público. Permite aproximar al alumnado a la idea de que el crecimiento de la ciudad se planifica (planeamiento urbano) o es fruto de acciones de reivindicación ciudadana.

- Comprobación de la existencia o no de equipamientos o servicios que condicionan el uso. Caracterización de los usos predominantes y reconocimiento de nuevos usos que se promueven desde estos espacios.

\section{Puesta en práctica de la actividad}

Introducción. Aporta información atractiva para el alumnado sobre el tema a investigar. El profesor/a mostrará la relevancia de los espacios públicos urbanos como lugares estructurantes en la trama urbana y de encuentro de personas y de socialización; y expondrá las características generales del espacio público elegido.

Tareas. Constituye el núcleo del trabajo a ejecutar. Los alumnos deben identificar las características físicas del espacio público elegido, el uso de dicho espacio por parte de los diversos grupos de usuarios y hacer propuestas de mejora.

\section{Procesos para realizar las tareas.}

a. Formación de los grupos de trabajo. La actividad se realizará en trabajo colaborativo, agrupándose los alumnos en grupos de cuatro a cinco miembros.

b. Explicación por parte del profesor del manejo de las fichas de trabajo. 
c. Observación del espacio elegido por parte de los grupos de trabajo acompañados por el profesor. En esta fase el docente les enseña a mirar el espacio: la ubicación del lugar en la trama urbana, las características del entorno, los componentes principales (cerramiento, mobiliario, dotaciones, jardines, etc.) desarrollando los conceptos y el vocabulario básico.

d. Recogida autónoma de la información por parte de los grupos mediante las fichas de trabajo y obtención de fotografías.

e. Tratamiento de la información: localización del espacio en el plano urbano; vaciado de los datos a partir del uso de un programa de hoja de cálculo (Excel); elaboración de gráficos; recopilación de imágenes y selección de aquellas que resultan más expresivas, etc.

f. Redacción de un informe que incluya las características físicas del espacio trabajado; el uso que los diversos grupos de ciudadanos hacen de él; la percepción que el grupo de trabajo tiene del espacio y las propuestas de mejora.

\section{Recursos}

a. Ficha de identificación del espacio público.

b. Ficha de uso del espacio público.

c. Planos.

d. Cámara fotográfica.

Fichas de Trabajo. El elemento básico para la realización de la actividad es la ficha de trabajo. Para facilitar la recogida de información y poder sistematizarla cada grupo completará dos tipos de fichas diferentes: una ficha de identificación del espacio y otra sobre el uso y las prácticas que los usuarios realizan.

La ficha de identificación está orientada a recopilar todos aquellos datos que permitirán hacer una descripción física del espacio público elegido y por tanto recogerá el nombre (oficial y/o popular), la localización dentro de la ciudad (barrio), la tipología (extensión, forma), las condiciones topográficas, la accesibilidad tanto externa (paradas de autobuses, tranvía, taxis) como interna (rampas, escaleras...), la pavimentación, la existencia de equipamientos interiores (parques infantiles, aseos, quioscos...), la descripción del mobiliario urbano (bancos, farolas...), la existencia de elementos de calidad ambiental (arbolado, jardines, fuentes, estanques...), la presencia de elementos artísticos, el estado de conservación, etc.

La ficha puede ser completada en el aula a partir de la utilización de planos digitales que facilitan su localización y la medición de su superficie.

La ficha de uso se encarga de recoger la información acerca de los usuarios del espacio público en estudio durante un tiempo predeterminado, al menos en dos horarios contrastados que permitan comprobar el uso real del espacio. En este caso, el alumnado anotará el número de usuarios, definirá si estos utilizan el lugar de forma individual o en grupo, se diferenciarán los usuarios por grupos de edad y sexo y se definirán las formas de uso más habituales (descanso, charla, paseo, lectura, juego, deporte...). En esta ficha, los alumnos podrán incluir otras anotaciones referidas a los usuarios (turistas, inmigrantes, indigentes...) que ayuden a una mejor comprensión de la apropiación del espacio público por parte de determinados colectivos y, también, relativas a las 
formas de estar y relacionarse (ocupación de áreas concretas, persistencia de los mismos grupos...). Es evidente que la recogida de información de esta segunda ficha exigirá a los alumnos no solo un ejercicio de observación sino también un esfuerzo de comunicación con los usuarios a fin de obtener una información clara y precisa.

Tanto la ficha de identificación como la de uso deben ir acompañadas de imágenes que ilustren cada uno de los datos o comentarios.

Puesta en común. Presentación en clase de los resultados obtenidos en formato Power-Point y debate posterior. La organización de la puesta en común de los resultados de aprendizaje debe programarse, de modo que cada uno de los grupos responda de sus tareas y pueda responder a las cuestiones principales relativas a los aspectos morfológicos y ambientales del espacio, a los usos más frecuentes y a la diversidad de usuarios. Para ello cada grupo deberá hacer una presentación al resto de compañeros en Power-Point. Una vez finalizadas las presentaciones, se inicia la discusión que, orientada por el profesor, debe abordar cuestiones centrales sobre la importancia de los espacios públicos abiertos para la calidad de vida de los habitantes urbanos, el valor de estos espacios como organizadores de la trama urbana, como contenedores de elementos ambientales, artísticos, lúdicos, etc. También se abordarán cuestiones relativas al género, a la edad y a la diversidad de los usuarios que se ha podido observar. El respeto por el otro, la conciencia de una ciudadanía plural y la necesidad de mejorar y conservar estos lugares de encuentro y relación deben ser abordadas en la puesta en común.

\section{Reflexiones finales}

Es evidente que los espacios públicos abiertos ofrecen a los docentes una interesante oportunidad como lugares de aprendizaje. Desde otras disciplinas, los espacios públicos abiertos han servido como vehículo didáctico, tal es el caso de las materias relativas a la educación ciudadana. También, desde las materias relacionadas con la Biología, la vegetación existente en parte de estos lugares ha permitido la realización de actividades formativas; la educación artística los ha utilizado bien como escenario de propuestas creativas o para analizar aquellos elementos artísticos que suelen exhibirse en ellos, bien de manera temporal o de forma permanente; la educación física aprovecha, muchas veces, el espacio abierto para realizar actividades o parte del mobiliario deportivo que ha ido apareciendo en muchas plazas y parques... En cambio, el espacio público ha sido muy poco utilizado como recurso didáctico para el aprendizaje geográfico a pesar de sus grandes potencialidades.

Analizando el currículum de Geografía, se constata que contiene temáticas que pueden ser trabajadas mediante actividades realizadas en estos lugares, donde el alumnado no solo aprenderá a descubrirlos como elementos estructurantes de la ciudad, sino a describirlos en sus aspectos morfológicos y funcionales esenciales. Podrá apreciar y valorar la diversidad de formas de usos que hace la población de estos lugares; la importancia de la conservación de los que ya existen y la necesidad de generar nuevos espacios que mejoren la calidad de vida de los habitantes de las ciudades. Además, servirá para reforzar gran parte de las competencias planteadas para los estudiantes de Secundaria y de Bachillerato.

La posibilidad de elegir entre una gran diversidad de espacio públi- 
cos, la relativa comodidad y seguridad que dispondrá el alumnado para trabajar en ellos, la capacidad formativa de estos lugares para lograr determinados aprendizajes transversales e involucrar a otras materias, $y$, sobre todo, la cercanía que cualquier espacio público transmite como espacio de la vida cotidiana, potencian las posibilidades del proceso de enseñanza-aprendizaje geográfico.

El esquema de propuesta didáctica que se ha planteado pretende ser una aproximación, desde la Geografía, ordenada en torno a dos ejes fundamentales: el espacio como elemento integrante de la ciudad, articulador de la trama urbana, contenedor de calidad ambiental, espacio de juego, paseo, etc. Y el espacio como lugar de relación, que admite una gran cantidad de usos y usuarios, un lugar colectivo que es usado y transformado según los distintos modos de apropiación y que es susceptible de rehabilitaciones, de renovaciones, que puede crearse de nuevo o eliminarse, y esto lo convierte, por su carácter público, en un elemento de gran interés para observar y analizar la evolución de la ciudad y la respuesta que esta da a los ciudadanos.

Se trata, en definitiva de leer la ciudad desde la propia experiencia, una óptica que va más allá de cualquier disciplina y que contribuye, como tantas otras acciones, al complejo aunque siempre alentador proceso de educar. 


\section{Referencias bibliográficas}

Benejam, P. (1999). El conocimiento científico y la didáctica de las ciencias sociales. Un curriculo de ciencias sociales para el siglo XXI. ¿Qué contenidos y por qué? Sevilla: Díada Editores, S. L.

Benayas, J.; Gutiérrez, J., y Gutiérrez, E. (1999). Educación ambiental en parques urbanos y espacios verdes análisis de una muestra de guías divulgativas y cuadernos didácticos. Tópicos en Educación Ambiental, vol.1 (1), 59-71. Recuperado el 10 de mayo de 2016, de: https:/ / dialnet.unirioja.es/servlet/ articulo? codigo=203596.

Borja, J. y Muxí, Z. (2000). Espacio público, ciudad y ciudadanía. Barcelona: Electa. Recuperado en 10 de mayo de 2010, de: http:/ / www.esdi-online.com/repositori/public/dossiers/DIDAC-wdw7ydy1.pdf

Borja, J. (2003). La ciudad conquistada. Madrid: Alianza Editorial. Recuperado el 12 de mayo de 2016, de: https:/ / derechoalaciudadflacso.files.wordpress. com/2014/01/jordi-borja-la-ciudad-conquistada. pdf.

Calero, C. G.; Delgado, C.R.; Ortiz, A. y Armas, A. (2014). Espacio público y género en áreas centrales renovadas: El Raval (Barcelona) y Cuatro Torres (Santa Cruz de Tenerife). Actas del XX Coloquio de Historia Canario Americana, 94-106. Recuperado el 10 de mayo de 2016, de: file:///C:/Users/usuario/ Downloads / 2014.\%20Calero, \%20Delgado, \% 20Ortiz, \%20\%20Armas\%20CHCA.pdf.

AGE (2007). Didáctica de la Geografía, 9. Madrid: Universidad Complutense de Madrid.

García de la Vega, A. (2008). Las claves metodológicas de un proyecto aplicado al proceso de enseñanza aprendizaje de la Geografía. Boletim Goiano de Geografia, 28 (1), 13-28.

García Ramón, M.D.; Ortiz, A. y Prats, M. (2004): Urban planning, gender and the use of public space in a peripherial neighbourhood of Barcelona Cities. International Journal of Urban Policy and Planning, 21 (3), 215-223.

Grupo de Pedagogía Urbana y Ambiental. (2008). Equidad y convivencia ciudadana en el espacio público. Bogotá: Universidad Pedagógica Nacional de Colombia

Godoy, I. y Sánchez, A. (2007). El trabajo de campo en la enseñanza de la Geografía. Sapiens. Revista Universitaria de Investigación, 8 (2), 137-146. Recuperado el 10 de mayo de 2016, de: http:/ / www.redalyc. org/pdf/410/41080209.pdf.

Martín Hernández, F. y Martín Ordoqui, J. (2009). La Geografía como campo educativo y de acción. Los desafíos y compromisos en el siglo XXI. Sapiens. Revista Universitaria de Investigación, 10 (1), 11-40.

Ministerio de Educación, Cultura y Deporte (2015a).
Real Decreto 1105/2014, de 26 de diciembre, por el que se establece el curriculo básico de la Educación Secundaria Obligatoria y del Bachillerato. BOE de 3 de enero de 2015. Recuperado el 19 de mayo de 2016, de: https://www.boe.es/boe/dias/2015/01/03/pdfs / BOE-A-2015-37.pdf.

Ministerio de Educación, Cultura y Deporte (2015b). Orden ECD/65/2015, de 21 de enero, por la que se describen las relaciones entre las competencias, los contenidos y los criterios de evaluación de la educación primaria, la educación secundaria obligatoria y el bachillerato. BOE de 29 de enero de 2015. Recuperado el 19 de mayo de 2016, de: https:/ / www.boe.es/diario-boe/txt. php?id=BOE-A-2015-738.

Molina, E. (2007). Escuela y educación fuera del aula: contribución de los escenarios exteriores al aprendizaje. Granada: Universidad de Granada. Revista Iberoamericana de Educación, 44, 4-10.

Montilla, A. (2005). El trabajo de campo: estrategia didáctica de la enseñanza de la Geografía. Geoenseñanza, 10 (2), 187-195. Recuperado el 10 de mayo de 2016, de: http://www.saber.ula.ve/bitstream/123456789/21019/2/articulo4.pdf.

Muñoz, J. M. y Olmos, S. (2010). Espacios abiertos y educación. Análisis e interpretación del lenguaje educativo de un espacio público. Revista de Educación, 352, 331-352. Recuperado el 10 de mayo de 2016, de: http:/ / www.revistaeducacion.mec.es/ re352-15.html.

Palacios, T. y Palacios, D. (1988). La indagación y la enseñanza de la Geografía en las Enseñanzas Medias. Anales de Geografía, 8, 59-72. Recuperado el 10 de mayo de 2016, de: http:/ / revistas. ucm.es/index.php/AGUC/article/viewFile/AGUC8888110059A/31915.

Páramo, P. (2010). Aprendizaje situado: creación y modificación de prácticas sociales en el espacio público urbano. Psicologia E Sociedade, 22 (1), 130-138. Recuperado el 10 de mayo de 2016, de: http:/ / www.scielo.br/pdf/psoc/v22n1/v22n1a16. pdf.

Perahia, R. (2007). Las ciudades y su espacio público. IX Congreso Internacional de Geocrítica. Los problemas del mundo actual: soluciones y alternativas desde la Geografía y las Ciencias Sociales. Recuperado en 10 de mayo de 2016, de: http://www.ub.edu/ geocrit/9porto/perahia.htm.

Priego González, C. (2004). El paisaje y los espacios públicos urbanos en el desarrollo de las sociedades. Centro Nacional de Educación Ambiental. Recuperado el 10 de mayo de 2016, de: http:/ / www.magrama.gob.es/es/ceneam/articulos-de-opinion/200405priego-tcm7-53039.pdf. 
Rodríguez, E. (2006). Enseñar geografía para los nuevos tiempos. Paradígma, 27 (2), 73-92. Recuperado el 10 de mayo de 2016, de: http:/ /www. scielo.org.ve/scielo.php?script=sci-arttext\&pid $=$ S1011-22512006000200005.

Souto, X. M. (1998). Didáctica de la Geografía. Problemas sociales y conocimiento del medio. Biblio 3W. Revista Bibliográfica de Geografía y Ciencias Sociales, 115 (5). Recuperado el 10 de mayo de 2016, de: http:/ / www.ub.edu/geocrit/b3w-115.htm.
Trilla, J. (2006). Introducción a la ciudad educadora. En Peiró i Gregòri, S. (coord.), Nuevos espacios y nuevos entornos de educación. Alicante: ECU, 19-42. Recuperado el 12 de mayo de 2016, de: http:/ / www.editorial-club-universitario.es/pdf/562.pdf.

Ucar, X. (2006). Investigación participativa sobre espacios públicos y educación cívica. Revista Iberoamericana de Educación, 39/4. Recuperado el 10 de mayo de 2016, de: http:/ / www.rieoei.org/experiencias132. htm.

DELGADO ACOSTA, Carmen Rosa, y Carmen, Gloria CALERO MARTÍN. Los espacios públicos urbanos: lugares para el aprendizaje geográfico. Hábitat y Sociedad, 2016, n. ${ }^{\circ}$ 9, pp. $157-174$.

$<w w w . h a b i t a t y s o c i e d a d . u s . e s>$

http://dx.doi.org/10.12795/HabitatySociedad.2016.i9.08

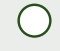

